\title{
Advanced choroidal melanoma with a desirable aesthetic outcome after enucleation: A case report
}

\author{
CHRYSSA TERZIDOU $^{1}$, ALEXANDRA TRIVLI ${ }^{1}$, GEORGIOS DALIANIS $^{1}$, \\ DIMITRA APESSOU ${ }^{2}$, DEMETRIOS A. SPANDIDOS ${ }^{3}$ and GEORGE N. GOULIELMOS ${ }^{4}$
}

\author{
Departments of ${ }^{1}$ Ophthalmology and ${ }^{2}$ Pathology, Konstantopouleio-Patission General Hospital, 14233 Nea Ionia, Athens; \\ ${ }^{3}$ Laboratory of Clinical Virology, Medical School, University of Crete, and ${ }^{4}$ Section of Molecular Pathology and \\ Human Genetics, Department of Internal Medicine, School of Medicine, University of Crete, 71003 Heraklion, Crete, Greece
}

Received April 9, 2018; Accepted May 8, 2018

DOI: $10.3892 / \mathrm{ol} .2018 .8661$

\begin{abstract}
Choroidal melanoma is a rare ocular tumor. The present study reports the case of a 66-year-old male who presented with chronic headache and progressive visual loss. Physical eye examination and combined A- and B-mode ultrasonography detected choroidal melanoma. Due to tumor characteristics the eye was enucleated restoring the orbital volume with a $22 \mathrm{~mm}$ intraorbital bioceramic sphere implant. The eye was subjected to histopathological examination that confirmed the choroidal melanoma, $2 \mathrm{~cm}$ in diameter and $0.8 \mathrm{~cm}$ in elevation, occupying almost half of the globe. Microscopically, the neoplasm comprises mostly of epithelioid cells and fewer Type B spindle cells, with intense pigmentation. AJCC staging for the melanoma was T4b. The patient was fitted with an artificial eye after enucleation. Thirteen months after initial diagnosis, liver metastases were confirmed during his scheduled follow-up.
\end{abstract}

\section{Introduction}

Choroidal melanoma is a rare neoplasm, although it is the most common primary intraocular malignant tumor in adults. The malignancy predominantly appears during the 6th decade of life. The malignancy arises at the uveal tract melanocytes and the choroid is most commonly involved. Clinical presentation is non-specific, depends on the location and size of the choroidal melanoma and includes blurring of vision, painless and progressive visual field loss, floaters and photopsia (1). Quite often the tumor is visible during fundoscopy, however, the most valuable diagnostic test is combined A- and B-mode ultrasonography (2). Prognosis depends on several factors including, age of the patient, tumor size, histological

Correspondence to: Dr Alexandra Trivli, Department of Ophthalmology, Konstantopouleio-Patission General Hospital, 3-5 Agias Olgas Str., 14233 Nea Ionia, Athens, Greece

E-mail: alextrivli@yahoo.com

Key words: choroidal, melanoma, ocular, enucleation, eye characteristics and the presence of metastases. Nonetheless, even with early diagnosis and appropriate treatment by using radiation or enucleation, an estimated $40-50 \%$ of all the patients eventually succumb to distal metastases $(3,4)$. In the present study, we report a case of choroidal melanoma, in a 66-year-old male. The aim of the current study is to demonstrate the importance of identifying a choroidal mass timely, in order to avoid possible metastases and to present the favorable aesthetic post-operative result.

\section{Case report}

In February 2017, a 66-year-old male presented to our clinic with chronic headache, photopsia and progressive vision loss of 11 months duration. In his history, the patient reported chronic obstructive pulmonary disease (COPD), five STEMIs (last one, one year prior) for which the patient was under antiplatelet therapy and cardiac arrhythmia for which he was administered amiodarone treatment. Complete ocular examination was performed: 7/10 cc right eye (RE) and 2/10 cc left eye (LE). Anterior segment was within normal in both eyes. Intraocular pressure was $15 \mathrm{mmHg}$ in both eyes. After mydriasis, fundoscopy of the left eye revealed a retinal detachment and underneath a solid dark gray mass in the nasal choroid. Fundoscopy of the right eye was unremarkable. B-scan ultrasound of the left eye was performed to assess the extent of the mass. A clinical diagnosis of choroidal melanoma was made based on our findings, and enucleation of the left eye was determined as the line of treatment.

Regarding surgery, conjunctival peritomy, isolation of the four rectus muscles, isolation and displacement of the oblique muscles and finally blunt approach to optic nerve was carried out with curved blunt end scissors in order to cut it as far as possible from the sclera. The Mesh-Wrapped Bioceramic Implant, a $22 \mathrm{~mm}$ diameter sphere covered with mesh (FCI ophthalmics), was implanted in the orbit to restore the volume and the recti muscles were attached in the appropriate position and secured at the mesh with 6/0 vicryl suture (Fig. 1). Tennon's capsule and conjunctiva were closed separately. A conformer was placed to cover the ocular surface and keep the fornixes in shape. Postoperative care was given systematically and topically. 


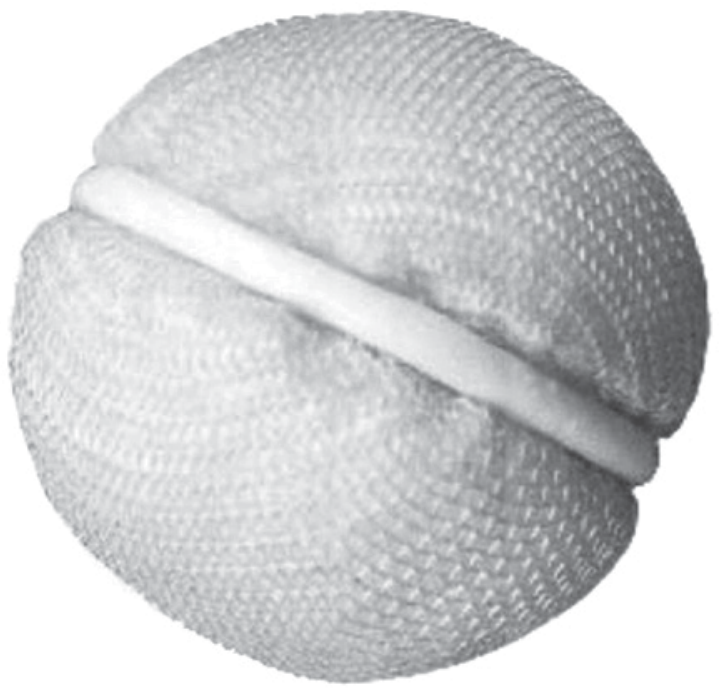

Figure 1. Mesh-wrapped bioceramic implant, used to restore the volume of the orbit.

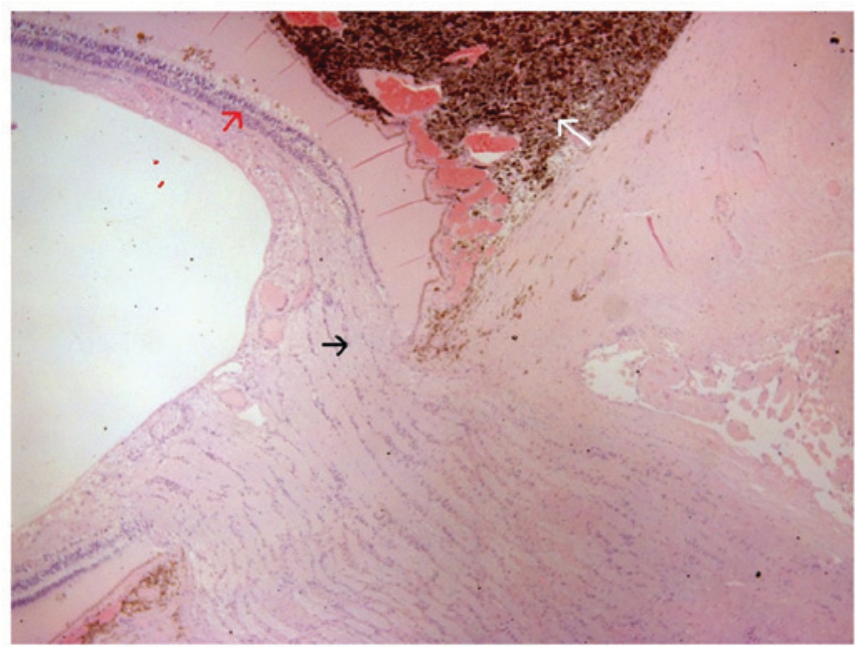

Figure 3. Choroidal melanoma near the optic nerve. Black arrow shows tumor near the optic nerve without infiltrating it; red arrow indicates the retina; white arrow indicates intense pigmentation.

The enucleated eye was subjected to histopathological examination. Histopathology report describes a choroidal melanoma $2 \mathrm{~cm}$ in diameter and $0.8 \mathrm{~cm}$ in elevation, occupying almost half of the globe, projecting in the vitreous cavity, detaching the retina and in contact with the ciliary body. Microscopically, the neoplasm comprised mostly of epithelioid cells and fewer Type B spindle cells, with intense pigmentation, no lymphocytic infiltration and no apparent necrosis, while it infiltrated the radial portion of the ciliary body (Fig. 2), the sclera and extended near the optic nerve without infiltrating it (Fig. 3). AJCC staging for the melanoma was T4b. After two months a smooth ocular surface was preset and a custom-made artificial eye was provided with excellent aesthetic result. A follow-up of 13 months shows unremarkable ocular examination; however, on the 14th month after initial diagnosis, oncology screening revealed several metastases in the liver.

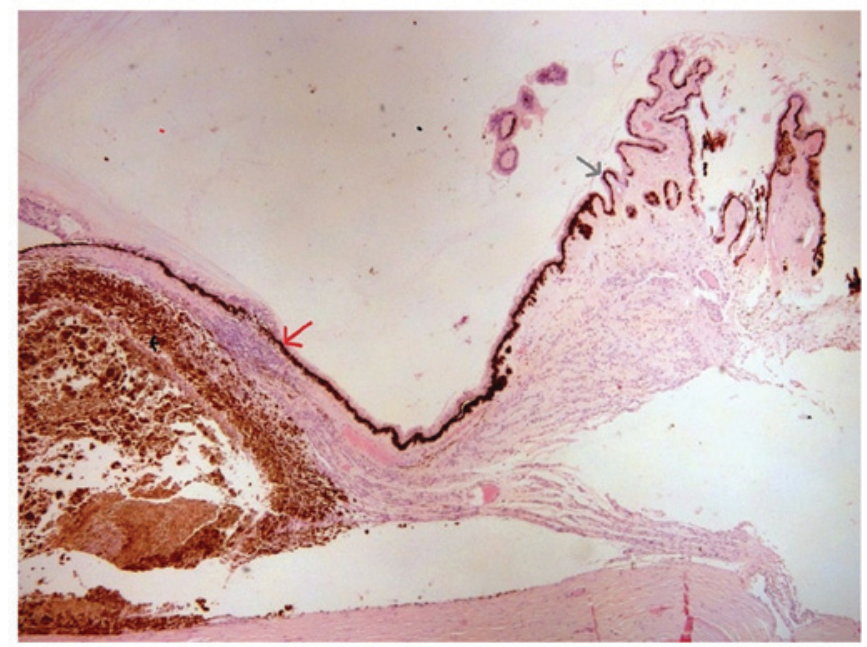

Figure 2. Choroidal melanoma. Grey arrow indicates ciliary body; red arrow shows infiltration of radial portion of ciliary body; black arrow shows the melanoma.

The Ethics Committee for Human Research of the Human Research of the Konstantopouleio-Patission General Hospital approved the study.

\section{Discussion}

Uveal melanoma is an uncommon entity. The estimated incidence of primary choroidal melanomas 6-7.5 cases per 1 million population with Caucasians most frequently affected. In most cases, melanomas arise at about 55 years; however they can occur at any age. These tumors are found slightly more frequently in men. Nonetheless, choroidal melanoma is the most common primary intra-ocular malignant tumor (1).

This malignancy arises from melanocytes in the choroid, ciliary body, or iris. The most common site involved is the choroid posterior to the equator, with approximately $85 \%$ of cases localized to this area (5). The pathophysiology of uveal melanoma, which includes choroidal and ciliary body tumors, remains enigmatic. Apart from the progress thus far regarding the establishment of some prognostic tools, the molecular prognostic information remains obscure. There is a well-known association of monosomy 3 and the development of aggressive uveal melanoma. More recently, specific abnormalities in loci associated with high-risk melanoma have been identified including $3 \mathrm{p}$ and $1 \mathrm{p}$ losses and 8q gain; however no common molecular pathway has yet been found (6).

Genetics studies have emphasized the role of specific mutations of GNAQ and GNA11 (members of large G proteins) as well as CDKN2A, BRCA2, p14/ARF and BAPI genes in the development of choroidal melanoma (7-10). A previous study, which focused on genetic factors associated with pigmentation traits, demonstrated the importance of rs12913832, rs1129038 and rs916977 polymorphisms of HERC2/OCA2 genomic region as susceptibility factors of uveal melanoma (11).

Next generation sequence performed by using uveal melanoma tumors indicated the significance of mutations of EIFIAX and SF3B1 genes as predisposition factors $(12,13)$. Furthermore, an RNA-based assay for choroidal melanoma prognosis that examines the expression profile of 15 genes 
has been developed aiming to generate prognostic subgroups for metastasis risk (14). Of note, comparative genomic hybridization failed to detect any defects or deletions when DNA from tumors was analyzed (15).

Suspected risk factors include iris color, skin color, hormonal influence and acute or intense exposure to ultraviolet light; however, host factors remain the prominent known risk factor with ancestry playing the strongest role $(16,17)$. The risk of choroidal and ciliary body melanomas in patients with nevi of the uveal tract is low, according to the available literature (18).

The clinical presentation of choroidal melanomas tends to vary as findings can be vague or non-specific and associated with the location of the tumor. Patients usually present with blurring of vision, painless and progressive visual field loss, floaters and photopsia. Ocular pain can present as the tumor presses on the ciliary nerves or due to acute angle closure glaucoma. However, frequently the patient remains asymptomatic until the mass has grown sufficiently to produce such symptoms. Notably, with the choroid layer being devoid of lymphatics, choroidal melanomas mostly metastasize via haemotogenous route mainly to the liver $(19,20)$.

The classic appearance of the neoplasm on dilated fundoscopy is a pigmented, dome or mushroom shaped tumor (indicating extension through Bruch's membrane) with surface vasculature and orange lipofuscin pigmentation, with an associated exudative retinal detachment (usually with melanomas greater than $4 \mathrm{~mm}$ in thickness). Notably, choroidal melanomas are usually pigmented, but their pigmentation can be vary and even be amelanotic. Findings that are not typical of choroidal melanomas and may indicate an alternative condition are drusen overlying the lesion, choroidal neovascularization over the surface of the tumor and bilateral observation of the lesion (21).

Combined A- and B-mode ultrasonography represent the most valuable diagnostic test. On A-scan ultrasonography, choroidal melanoma shows medium to low internal echoes with smooth attenuation and usually visible vascular pulsations. On B-scan ultrasonography, an acoustically silent zone within the melanoma, choroidal excavation and shadowing in the orbit are classically observed. For tumors greater than $3 \mathrm{~mm}$ in thickness, a combination of A and B-scan ultrasonography has $95 \%$ accuracy in choroidal melanomas diagnosis (2).

Fluorescein angiography is helpful in identifying features suspicious for melanoma, including areas of fluorescein dye leakage and intrinsic tumor circulation (double circulation) located in and around the lesion (3).

The overall prognosis of this rare entity is based on several factors: The patient's age, tumor size, histological characteristics and the presence of metastasis (3). However, even with early diagnosis and appropriate treatment by using radiation or enucleation and follow-up, it is estimated that $40-50 \%$ of all patients will eventually die due to distal metastases (4).

Management depends on tumor size and targets maximum vision sparing, quality of life and emotional result, considering that enucleation is a form of amputation for the patient. For this reason, important and of great consideration is the postoperative restoration of the appearance of the enucleated orbit. The use of new materials of intraorbital implants to restore orbit volume, that allow suturing of the muscles on them while minimizing complications of extrusion and inflammation of the implant, provides implant motility and a good infrastructure for fitting custom made artificial eyes with excellent aesthetic result, eliminating one of the emotional stresses of these patients.

Metastases and general health status should be considered in the treatment decision. Enucleation tends to be the method usually preferred for medium and large ocular melanomas, considered primarily in cases of diffuse melanomas and in the presence of extraocular extension. Other published techniques described, are plaque brachytherapy with iodine-125, gold-198 or palladium-103, external beam irradiation with charged particles, protons, or helium nuclei and Gamma knife surgery. Eyewall resection or sclerouvectomy is an alternative option proposed in the literature (22).

In conclusion, although uveal melanomas are rare, one should be cautious when examining a choroidal mass. The patient should be informed about possible metastases, living expectancy, treatment options and expected vision outcome. Early detection is important, thus fundus examination should be considered in patients over 40 when they are routinely examined for presbyopia.

\section{Acknowledgements}

We would like to thank all the clinicians for providing the data used in this study.

\section{Funding}

No funding was received.

\section{Availability of data and materials}

All data generated or analyzed during this study are included in this published article.

\section{Authors' contributions}

CT was the primary surgeon, who designed and performed the surgical procedure. GD was the assistant surgeon. AT designed the study and wrote the manuscript. GNG, GD, DA and DAS collected the data and critically revised the manuscript.

\section{Ethics approval and consent to participate}

The Ethics Committee for Human Research of the Human Research of the Konstantopouleio-Patission General Hospital approved the study.

\section{Consent for publication}

The patient provided written informed consent for the publication of any associated data and accompanying images.

\section{Competing interests}

Demetrios A. Spandidos is the Editor-in-Chief for the journal, but had no personal involvement in the reviewing process, or any influence in terms of adjudicating on the final decision, for this article. 


\section{References}

1. Rodríguez A, Dueñas-Gonzalez A and Delgado-Pelayo S: Clinical presentation and management of uveal melanoma. Mol Clin Oncol 5: 675-677, 2016.

2. Char DH, Stone RD, Irvine AR, Crawford JB, Hilton GF, Lonn LI and Schwartz A: Diagnostic modalities in choroidal melanoma. Am J Ophthalmol 89: 223-230, 1980.

3. Kanski J and Bowling B: Clinical Ophthalmology: A Systematic Approach (7th ed). Elsevier/Saunders, New York, pp501-504, 2011.

4. Kujala E, Makitie T and Kivela T: Very long-term prognosis of patients with malignant uveal melanoma. Invest Ophthalmol Visual Sci 44: 4651-4659, 2003.

5. Shields CL, Kaliki S, Furuta M, Mashayekhi A and Shields JA: Clinical spectrum and prognosis of uveal melanoma based on age at presentation in 8,033 cases. Retina 32: 1363-1372, 2012.

6. Sisley K, Rennie IG, Parsons MA, Jacques R, Hammond DW, Bell SM, Potter AM and Rees RC: Abnormalities of chromosomes 3 and 8 in posterior uveal melanoma correlate with prognosis. Genes Chromosomes Cancer 19: 22-28, 1997.

7. Van Raamsdonk CD, Griewank KG, Crosby MB, Garrido MC, Vemula S, Wiesner T, Obenauf AC, Wackernagel W, Green G, Bouvier N, et al: Mutations in GNA11 in uveal melanoma. N Engl J Med 363: 2191-2199, 2010.

8. Van Raamsdonk CD, Bezrookove V, Green G, Bauer J, Gaugler L, O'Brien JM, Simpson EM, Barsh GS and Bastian BC: Frequent somatic mutations of GNAQ in uveal melanoma and blue naevi. Nature 457: 599-602, 2009.

9. Buecher B, Gauthier-Villars M, Desjardins L, Lumbroso-Le Rouic L, Levy C, De Pauw A, Bombled J, Tirapo C, Houdayer C, Bressac-de Paillerets $\mathrm{B}$, et al: Contribution of $\mathrm{CDKN}_{2} \mathrm{~A} / \mathrm{P} 16$ (INK4A), P14 (ARF), $\mathrm{CDK}_{4}$ and $\mathrm{BRCA}_{1 / 2}$ germline mutations in individuals with suspected genetic predisposition to uveal melanoma. Fam Cancer 9: 663-667, 2010.

10. Abdel-Rahman MH, Pilarski R, Cebulla CM, Massengill JB, Christopher BN, Boru G, Hovland P and Davidorf FH: Germline BAP1 mutation predisposes to uveal melanoma, lung adenocarcinoma, meningioma, and other cancers. J Med Genet 48 : 856-859, 2011.

11. Ferguson R, Vogelsang M, Ucisik-Akkaya E, Rai K, Pilarski R, Martinez CN, Rendleman J, Kazlow E, Nagdimov K, Osman I, et al: Genetic markers of pigmentation are novel risk loci for uveal melanoma. Sci Rep 6: 31191, 2016. doi:10.1038/srep31191.

12. Harbour JW, Roberson ED, Anbunathan H, Onken MD, Worley LA and Bowcock AM: Recurrent mutations at codon 625 of the splicing factor $S F 3 B 1$ in uveal melanoma. Nat Genet 45: 133-135, 2013.
13. Martin M, Maßhöfer L, Temming P, Rahmann S, Metz C, Bornfeld N, van de Nes J, Klein-Hitpass L, Hinnebusch AG, Horsthemke B, et al. Exome sequencing identifies recurrent somatic mutations in EIFIAX and SF3B1 in uveal melanoma with disomy 3. Nat Genet 45: 933-936, 2013.

14. Onken MD, Worley LA, Char DH, Augsburger JJ, Correa ZM, Nudleman E, Aaberg TM Jr, Altaweel MM, Bardenstein DS, Finger PT, et al: Collaborative Ocular Oncology Group report number 1: Prospective validation of a multi-gene prognostic assay in uveal melanoma. Ophthalmology 119: 1596-1603, 2012.

15. Speicher MR, Prescher G, du Manoir S, Jauch A, Horsthemke B, Bornfeld N, Becher R and Cremer T: Chromosomal gains and losses in uveal melanomas detected by comparative genomic hybridization. Cancer Res 54: 3817-3823, 1994.

16. Weis E, Shah CP, Lajous M, Shields JA, Shields CL: The association between host susceptibility factors and uveal melanoma: A meta-analysis. Arch Ophthalmol 124: 54-60, 2006.

17. Seddon JM, Gragoudas ES, Glynn RJ, Egan KM, Albert DM and Blitzer PH: Host Factors, UV radiation, andrisk of uveal melanoma. Arch Ophthalmol 108: 1274-1280, 1990.

18. Smith JH, Padnick-Silver L, Newlin A, Rhodes K and Rubinstein WS: Genetic study of familial uveal melanoma: Association of uveal and cutaneous melanoma with cutaneous and ocular nevi. Ophthalmology 114: 774-779, 2007.

19. Kath R, Hayungs J, Bornfeld N, Sauerwein W, Hoffken K and Seeber S: Prognosis and treatment of disseminated uveal melanoma. Cancer 72: 2219-2223, 1993.

20. Shields C, Materin M, Shields J, Gershenbaum E, Singh A and Smith A: Factors associated with elevated intraocular pressure in eyes with iris melanoma. Br J Ophthalmol 85: 666-669, 2001.

21. Kanski J: Signs in Ophthalmology: Causes and differential diagnosis. 66824th edition. Elsevier, New York, NY, pp 310-318, 2010.

22. Singh P and Singh A: Choroidal melanoma. Oman J Ophthalmol 5: 3-9, 2012.

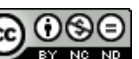

This work is licensed under a Creative Commons Attribution-NonCommercial-NoDerivatives 4.0 International (CC BY-NC-ND 4.0) License. 